\title{
PERSPECTIVE
}

\section{Managing Chronic Pain in Primary Care: It Really Does Take a Village}

\author{
Karen Seal, MD, MPH ${ }^{1,2}$, William Becker, $\mathrm{MD}^{3,4}$, Jennifer Tighe, $M S P H^{7}$, Yongmei Li, $P h D^{7}$, \\ and Tessa Rife, PharmD, CACP, CGP

\begin{abstract}
${ }^{1}$ San Francisco VA Healthcare System, San Francisco, CA, USA; ${ }^{2}$ University of California, San Francisco, San Francisco, CA, USA; ${ }^{3}$ VA Connecticut
\end{abstract} \\ Healthcare System, West Haven, CT, USA; ${ }^{4}$ Yale University School of Medicine, New Haven, CT, USA.
}

Some healthcare systems are relieving primary care providers (PCPs) of "the burden" of managing chronic pain and opioid prescribing, instead offloading chronic pain management to pain specialists. Last year the Centers for Disease Control and Prevention recommended a biopsychosocial approach to pain management that discourages opioid use and promotes exercise therapy, cognitive behavioral therapy and non-opioid medications as first-line patient-centered, multi-modal treatments best delivered by an interdisciplinary team. In the private sector, interdisciplinary pain management services are challenging to assemble, separate from primary care and not typically reimbursed. In contrast, in a fully integrated health care system like the Veterans Health Administration (VHA), interdisciplinary clinics already exist, and one such clinic, the Integrated Pain Team (IPT) clinic, integrates and co-locates pain-trained PCPs, a psychologist and a pharmacist in primary care. The IPT clinic has demonstrated significant success in opioid risk reduction. Unfortunately, proposed legislation threatens to dismantle aspects of the VA such that these interdisciplinary services may be eliminated. This Perspective explains why it is critical not only to maintain interdisciplinary pain services in VHA, but also to consider disseminating this model to other health care systems in order to implement patient-centered, guideline-concordant care more broadly.

KEY WORDS: chronic pain; opioids; interdisciplinary care; primary care.

J Gen Intern Med 32(8):931-4

DOI: $10.1007 / \mathrm{s} 11606-017-4047-5$

(c) Society of General Internal Medicine 2017

I n the spring of 2016, The New York Times featured an article on primary care clinics in West Virginia that had hired an anesthesiologist to treat patients with chronic pain outside of primary care, thus "relieving its primary care doctors and nurse practitioners of their thorniest burden." The article lauded the anesthesiologist/pain specialist, who is quoted as

Received September 2, 2016

Revised December 30, 2016

Accepted March 9, 2017

Published online March 23, 2017 saying, "I'm part F.B.I. investigator, part C.I.A. interrogator, part drill sergeant, part cheerleader."1

Is treating chronic pain no longer the job of primary care providers (PCPs)? Chronic pain complaints are the second most common reason for outpatient primary care visits: over 100 million Americans suffer from chronic pain, more than the total number affected by heart disease, cancer and diabetes combined. Painful conditions also burden the U.S. economy, costing \$635 billion each year in medical treatment and lost productivity. ${ }^{2}$ Yet, PCPs report discomfort in managing chronic pain, especially given mounting pressure to curtail patients' use of opioids. ${ }^{3}$ Perhaps chronic pain management actually has become one of primary care's thorniest burdens. After all, PCPs did not sign up to become FBI investigators, CIA interrogators, drill sergeants or cheerleaders-well, maybe cheerleaders....

At a time when patients are dying in record numbers from opioids prescribed for pain, perhaps PCPs should offload chronic pain management to specialists, as The Times article suggests. Yet this would risk moving backwards toward an antiquated biomedical model of specialty pain treatment, paradoxically the same model that got us into this mess in the first place. The biomedical model assumes a singular biological cause for chronic pain; we just need a diagnosis and then we can "cure" the pain. This sets both provider and patient on a crash course of diagnostic testing, polypharmacy and procedures. In the U.S., hundreds of billions of dollars are spent on specialty procedures and opioid pain medications for chronic pain, and while these therapies may be appropriate in certain settings, outcomes for chronic pain are not well substantiated. On the contrary, at least in the case of chronic opioid therapy, there is growing evidence of serious harm. ${ }^{4}$

In contrast, the 2011 Institute of Medicine's consensus report, the Centers for Disease Control and Prevention (CDC) Guideline for Prescribing Opioids for Chronic Pain (2016) and the Department of Health and Human Services' National Pain Strategy (2016) have all called for a biopsychosocial approach in the treatment of chronic pain. This radically shifts the focus away from "fixing" pain, toward improving the experience of individuals with chronic pain and enhancing functioning and quality of life. ${ }^{2,5,6}$ Similarly, the National Pain Strategy calls for an evidence-based multimodal and interdisciplinary treatment approach to chronic pain. ${ }^{5}$ 
The CDC Guideline specifically recommends avoiding the use of opioids in favor of exercise therapy, cognitive behavioral therapy and non-opioid medications as first-line treatments for chronic pain. ${ }^{6}$ But how is an individual PCP, or even a specialist, expected to implement guidelines that essentially call for a team-based approach to chronic pain? What insurance plan reimburses this?

In fact, the Veterans Health Administration (VHA), the largest publicly funded, fully integrated health care system in the U.S., has been a leader in addressing these gaps in implementation. In 2009, the VHA introduced a Stepped Care framework in which patients receive biopsychosocial chronic pain care first within VA primary care, instead of routinely triaging patients with chronic pain to specialists. Since 2009, interdisciplinary pain clinics have been implemented at a few VA medical centers throughout the country. These interdisciplinary clinics co-locate and integrate PCPs, psychologists, pharmacists and/or physical therapists to provide multimodal pain care, often directly in primary care, and preliminary results are promising. ${ }^{7}$

In July of 2015, the Integrated Pain Team (IPT) was established within primary care in the San Francisco VA Health Care System (SFVAHCS). The mission of the IPT is to provide focused interdisciplinary, biopsychosocial pain care that also addresses the psychological comorbidities such as posttraumatic stress disorder (PTSD) and depression that are prevalent among patients with chronic pain, particularly veterans; these same patients have also been found to have higher rates of opioid use and misuse. ${ }^{8}$ The interdisciplinary IPT clinic integrates and co-locates pain-trained PCPs with a psychologist and pharmacist with expedited access to physical and recreational therapy. Other PCPs are encouraged to refer patients with complex chronic pain, irrespective of whether they are prescribed opioids, and over the course of weeks to months, IPT providers assume responsibility for pain care, including prescribing. Specifically, IPT providers collaborate with the patient and the referring PCP to develop a multimodal pain care plan that emphasizes the use of exercise, behavioral interventions and complementary and integrative modalities, while reducing reliance on pain medications, especially opioids. On patient discharge, IPT providers conduct a warm handoff back to the referring PCP, which consists of detailed in-person or electronic recommendations intended to empower PCPs to assume long-term management of their patients' chronic pain issues. In fact, one important function of the IPT in primary care is to provide pain management education for other PCPs in the form of these recommendations as well as regular educational sessions, all of which is intended to increase PCPs' self-efficacy in managing complex chronic pain complaints. The IPT is physically located at the San Francisco VA Medical Center, where local patients may be seen in person. The San Francisco-based team also conducts virtual interdisciplinary pain management visits for patients based at more rural VHA community clinics throughout northern California, using video telehealth technology.
As a case example, a PCP with a patient who has chronic pain and is co-prescribed high-dose opioids for pain and benzodiazepines for an anxiety disorder may refer the patient to the IPT. IPT providers will engage in shared decisionmaking with the patient to develop a Pain Care Plan aligned with the patient's personal values and goals, which might include strategies such as gentle exercise, yoga or another enjoyable hobby (e.g., gardening), cognitive behavioral therapy for pain, and non-opioid analgesics to reduce reliance on opioids. At the same time, IPT providers may attempt to reduce opioid overdose risk by tapering either the opioid or the benzodiazepine, or both, perhaps suggesting a nonbenzodiazepine medication to treat anxiety symptoms. On discharge, IPT providers will share the patient's Pain Care Plan with the referring PCP to promote continuity of care.

Of note, while there are numerous positive randomized controlled trials for chronic pain using cognitive behavioral therapy ${ }^{9,10}$ and exercise, ${ }^{11,12}$ to date, there has not been a comparative effectiveness trial evaluating the biopsychosocial IPT model against other models of pain care delivery (e.g., pharmacist collaborative pain management). Moreover, there are no studies that evaluate the effect of an interdisciplinary biopsychosocial approach on neurohormones (e.g., cortisol), that may mediate the putative positive effects on chronic pain and mental health comorbidities such as anxiety that can exacerbate and perpetuate chronic pain.

To begin to fill this research gap, we have been conducting a quality improvement study of the IPT clinic based at the SFVAHCS. Preliminarily, we examined 162 veteran patients: 81 patients enrolled in the IPT clinic compared to 81 matched control patients who were seen in usual primary care during the same time period. We followed both groups for 90 days. All patients had chronic pain, were prescribed opioids and were matched with respect to age, sex, number and type of psychiatric diagnoses, and baseline daily opioid dose. We found that the mean daily opioid dose (measured by morphine equivalent daily dose [MEDD]) decreased by $41.2 \mathrm{mg}$ in the IPT group after 90 days (from 108.7 to $67.6 \mathrm{mg}$ ), while the reduction in the control group was only $24.8 \mathrm{mg}$ ( 102.3 to $95.5 \mathrm{mg}$ ). Twice as many patients in the IPT clinic as in the control group (38 vs. 19 patients) reduced their daily opioid dose by $50 \%$ or more, representing a 2.6 -fold increase in the odds of a $50 \%$ or greater reduction in daily opioid use $(p=0.004)$. With follow-up extended to 180 days, the daily prescription opioid dose of the IPT group was $103 \%$ lower than that of the control group $(p=0.015)$; receiving care through the IPT clinic was associated with 3.6-fold increased odds of $50 \%$ or greater reduction in opioid use $(p=0.005)$. In sum, these preliminary quality improvement data suggest that interdisciplinary team-based pain care in collaboration with primary care may be superior to usual primary care alone in reducing reliance on opioids for pain management. This, in turn, may lead to greater reductions in patient harm, given the increased risk for opioid overdose associated with higher doses of opioids. 
Regrettably, though, this groundbreaking interdisciplinary pain care model may be jeopardized by recent political developments, including recent election results. In addition, several bills introduced in Congress (e.g., the Permanent Department of Veterans Affairs Choice Card Act of 2015, the Veterans Health Care Freedom Act, 2015), along with recommendations of the 2016 Commission on Care report, ${ }^{13}$ propose to channel the majority of veterans' medical care (including pain care) to the private sector, where coordinated treatment is not the norm. While creating limited networks and partnerships with private sector providers and organizations has the potential to enhance veterans' options and access to care, the passage of these bills should not come at a cost of defunding and hence undermining the kind of advances our team-based approach demonstrates. This would represent a disappointing step backwards given the wealth of evidence showing that the VHA outperforms private sector healthcare in outpatient care on almost all quality measures. ${ }^{14}$ The Integrated Pain Team clinic is one example of what's at stake; these hardwon gains in interdisciplinary health services merit further support and dissemination throughout VA primary care and even to other healthcare systems. As with chronic pain, lives may depend on coordinated team-based management and safe prescribing.

In fact, primary care-based integrated pain teams like those in the VA are rare in the private sector, mostly because biopsychosocial pain care is time-intensive and not reimbursed at the same high rate as specialty pain care, which commonly emphasizes procedures. Interdisciplinary teambased care, while relatively easy to assemble in a fully integrated health system like the VA, is far less feasible in a more fragmented private sector healthcare system. Yes, interdisciplinary team-based care takes more time up front than simply prescribing a pill, but in the long run, improved safety and efficacy of a team-based, opioid-avoidance approach may produce benefits for individual patients and populations alike. ${ }^{15}$ Moreover, team-based chronic pain care delivered in a primary care setting is consistent with the evidence-based, multi-modal strategies advanced by the CDC and other national health care agencies to reduce reliance on opioids and improve safe opioid prescribing. In keeping with the CDC and other national guidelines, ancillary primary care staff in some VA centers assist in aligning pain care planning with patient values and goals and providing psychotherapy, physical rehabilitation and pharmacy support, ultimately exceeding what any individual PCP could provide.

In sum, many well-intentioned efforts to relieve chronic pain through specialty care have made people sicker, primarily by increasing over-reliance on opioids, further contributing to fragmented healthcare and soaring costs. PCPs are best positioned to deliver biopsychosocial chronic pain care: they have long-term relationships with their patients and can consider patients' values and goals in developing patient-centered pain care plans. Nevertheless, PCPs need ancillary team support - "a village" - to implement this guideline-concordant care. As a publicly funded health care system swimming upstream against a current toward privatization, the VA continues to pave the way toward primary care-based pain management, which is biopsychosocial in orientation and patient-centered. Providing interdisciplinary support to PCPs is critical to delivering highquality chronic pain care in primary care, so that it never becomes PCPs" "thorniest burden."

\begin{abstract}
Acknowledgements:
Contributors: The results reported in this commentary would not be possible without the hard work of the Integrated Pain Team Clinic staff at the San Francisco VA Healthcare System, whose members include: Sarah Palyo, PhD; Erin Watson, PhD; Payal Marpara, PhD; Caitlin Garvey, NP; Christina Tat, PharmD, BCPS; and Elizabeth Son, PharmD. We wish to thank Thomas Metzler, MS, for assisting with statistics, and Ana-Marie Urbieta for her former military service and work on this study. We also acknowledge Russell Lemle, $P h D$, and Suzanne Gordon for their contributions to this perspective.
\end{abstract}

Publisher's Note: Springer Nature remains neutral with regard to jurisdictional claims in published maps and institutional affiliations.

Corresponding Author: Karen Seal, MD, MPH; San Francisco VA Healthcare System, Box 116-A, 4150 Clement Street, San Francisco, CA 94121, USA (e-mail: karen.seal@ucsf.edu; karen.seal@va.gov).

\section{Compliance with Ethical Standards:}

Funders: Preliminary research reported in this commentary is funded by a Department of Veterans Affairs Quality Enhancement Research Initiative (QUERI) award (Mary Whooley, MD, Principal Investigator). The views expressed are those of the authors only and do not reflect the opinions of the Veterans Health Administration.

Conflict of Interest: The authors declare that they do not have a conflict of interest.

\section{REFERENCES}

1. Goodnough A. Treating pain without feeding addiction at epicenter for opioids. The New York Times. May 11, 2016.

2. Institute of Medicine (US) Committee on Advancing Pain Research, Care, and Education. Relieving Pain in America: A Blueprint for Transforming Prevention, Care, Education, and Research. Washington (DC): National Academies Press (US); 2011. http:// www.nationalacademies.org/hmd/Reports/2011/Relieving-Pain-inAmerica-A-Blueprint-for-Transforming-Prevention-Care-EducationResearch.aspx. Accessed March 7, 2017.

3. Jamison RN, Sheehan KA, Scanlan E, Matthews M, Ross EL. Beliefs and attitudes about opioid prescribing and chronic pain management: survey of primary care providers. J Opioid Manag. 2014;10(6):375-382.

4. Dunn KM, Saunders KW, Rutter CM, et al. Opioid prescriptions for chronic pain and overdose: a cohort study. Ann Intern Med. 2010;152(2):85-92.

5. United States Department of Health and Human Services Interagency Pain Research Coordinating Committee. National Pain Strategy: A Comprehensive Population Health-Level Strategy for Pain. March 2016. https://iprcc.nih.gov/National_Pain_Strategy/NPS_Main.htm. Accessed March 7, 2017.

6. Centers for Disease Control and Prevention. CDC Guideline for Prescribing Opioids for Chronic Pain - United States, MMWR: Recommendations and Reports. March 18, 2016; 65(1):1-49.

7. Dorflinger LM, Ruser C, Sellinger J, Edens EL, Kerns RD, Becker WC. Integrating interdisciplinary pain management into primary care: development and implementation of a novel clinical program. Pain Med. 2014;15(12):2046-2054.

8. Seal KH, Shi Y, Cohen G, Cohen BE, Maguen S, Krebs EE, Neylan TC. Association of mental health disorders with prescription opioids and 
high-risk opioid use in US veterans of Iraq and Afghanistan. JAMA. 2012;307(9):940-947.

9. Cherkin DC, Sherman KJ, Balderson BH, Cook AJ, Anderson ML Hawkes RJ, Hansen KE, Turner JA. Effect of mindfulness-based stress reduction vs cognitive behavioral therapy or usual care on back pain and functional limitations in adults with chronic low back pain: A randomized clinical trial. JAMA. 2016;315(12):1240-9.

10. Ehde DM, Dillworth TM, Turner JA. Cognitive-behavioral therapy for individuals with chronic pain: efficacy, innovations, and directions for research. American Psychologist. 2014;69(2): 153.

11. Searle A, Spink M, Ho A, Chuter V. Exercise interventions for the treatment of chronic low back pain: A systematic review and metaanalysis of randomised controlled trials. Clinical Rehabilitation. 2015;29(12):1155-67.
12. Wang SY, Olson-Kellogg B, Shamliyan TA, Choi JY, Ramakrishnan R, Kane RL. Physical therapy interventions for knee pain secondary to osteoarthritis: a systematic review. Ann Intern Med. 2012;157(9):632-44.

13. Final Report of the Commission on Care. Washington D.C., June 30, 2013. 2017 https://commissiononcare.sites.usa.gov/files/2016/07/ Commission-on-Care_Final-Report_063016_FOR-WEB.pdf. Accessed March 7, 2017.

14. RAND Health. (2015). Resources and capabilities of the Department of Veterans Affairs to provide timely and accessible care to veterans. Santa Monica, CA. Retrieved from http://www.rand.org/pubs/research_reports/RR1165z2.html. Accessed March 7, 2017.

15. Bair MJ, Ang D, Wu J, et al. Evaluation of Stepped Care for Chronic Pain (ESCAPE) in Veterans of the Iraq and Afghanistan Conflicts: A Randomized Clinical Trial. JAMA Intern Med. 2015;175(5):682-689. 